\title{
A NOTE ON LOGARITHMIC SUMMABILITY (L)
}

\author{
by Y. SITARAMAN
}

(Received 17th September 1964)

\section{Introduction}

It is well known that two mutually related summability methods for a sequence $s_{n}(n=0,1,2, \ldots)$ are any Cesaro method $(C, \gamma)$ of order $\gamma>0$ and the Abel method $(A)$. The notation used in this statement is that of Hardy ((1), pp. 96-7, 71) and the statement itself can be amplified as follows. Summability $(C, \gamma), \gamma>0$, of $s_{n}$ implies (i) $s_{n}=o\left(n^{\gamma}\right)$, (ii) summability $(A)$ of $s_{n}$. Also, as a conditional converse of this result, we have Offord's result ((3), first part of Theorem 2), that hypothesis (i), and hypothesis (ii) suitably strengthened, together imply summability $(C, \gamma), \gamma>0$, of $s_{n}$. It is the object of this note to bring to light a second pair of summability methods mutually related like the methods $(C, \gamma)$ and $(A)$, by following an argument which is essentially similar to Offord's but differs sufficiently from Offord's in details to justify a separate and self-contained treatment of the second pair of methods.

In the notation of two earlier papers by the present author and others (2), (4), this second pair of methods, called the logarithmic methods $(l)$ and $(L)$, is defined as follows. A sequence $s_{n}$ is summable $(l)$ to sum $S$, or briefly,

$$
\text { if } \left.\quad\left(s_{0}+\frac{s_{1}}{2}+\ldots+\frac{s_{n}}{n+1}\right) / \log (n+1) \rightarrow S(n \rightarrow \infty) ;\right\}
$$

while the sequence is summable $(L)$ to $S$, or,

$$
\left.\begin{array}{c}
s_{n} \rightarrow S(L), \\
\text { if } \quad\left(s_{0} x+\frac{s_{1}}{2} x^{2}+\ldots+\frac{s_{n}}{n+1} x^{n+1}+\ldots\right) / \log (1-x)^{-1} \rightarrow S(x \rightarrow 1-0),
\end{array}\right\}
$$

the numerator of the left-hand member of the last relation being a power series convergent (absolutely) for $|x|<1$. In more general circumstances, when the sequence tending to $S$ in (1) is merely bounded, we write

$$
s_{n}=O(1)(l)
$$

and, similarly, when the function tending to $S$ in (2) is just bounded, we write

$$
s_{n}=O(1)(L) \text {. }
$$

In connection with definitions (1) and (2) the following facts are known ((1), 
p. 87; (2), Theorem 3, 4). The method of summability $(l)$ is equivalent to the Riesz method $(R, \log (n+1), 1)$ in Hardy's notation. Also, summability $(l)$ of $s_{n}$ to sum $S$ implies (i) $s_{n}=o(n \log n)$, (ii) $s_{n} \rightarrow S(L)$. The purpose of the present note is, briefly, to obtain a result (Theorem 2) in which hypothesis (i), and hypothesis (ii) suitably augmented, together make $s_{n}$ summable $(l)$ to $S$. The augmented hypothesis (ii) may be related to a strengthened form of summability $(L)$ defined as follows in analogy with a strengthened form of summability $(A)$ discussed by Hardy ((1), p. 381).

Suppose that $s_{n}(n=0,1,2, \ldots)$ is such that

$$
F(z)=s_{0} z+\frac{s_{1}}{2} z^{2}+\ldots+\frac{s_{n}}{n+1} z^{n+1}+\ldots
$$

is a power series with circle of convergence $|z|=1$, and further

$$
\frac{F(z)}{\log (1-z)^{-1}} \rightarrow S \text { uniformly when } z \rightarrow 1
$$

subject to the condition

$$
|z|<1,-\frac{1}{2} \pi<\alpha \leqq \text { am }(1-z) \leqq \beta<\frac{1}{2} \pi .
$$

Then we write

$$
s_{n} \rightarrow S(L, \alpha, \beta),
$$

so that, in the particular case $\alpha=\beta=0$, the last relation becomes $s_{n} \rightarrow S(L)$. Similarly we write

if

$$
\left.\begin{array}{c}
s_{n}=O(1)(L, \alpha, \beta) \\
\frac{F(z)}{\log (1-z)^{-1}}=O(1) \text { uniformly when } z \rightarrow 1 \text { as in (3). }
\end{array}\right\}
$$

In virtue of a theorem of Montel's quoted by Offord ((3), p. 475), we see from definitions (3) and (4) that

$$
s_{n} \rightarrow S(L) \text { and } s_{n}=O(1)(L, \alpha, \beta) \text { together imply } s_{n} \rightarrow S(L, \alpha, \beta) \text {. }
$$

Theorem 1 of this note contains implicitly the main result in Theorem 2 and embodies a technique used by Offord to treat $(A)$ summability ((3), Theorem 1) with the necessary modification which makes it applicable to $(L)$ summability. Theorem 1 is preceded by lemmas which mark certain stages in its proof. In the proofs of the theorems and the lemmas, $K, K^{\prime}, M, N$ each denote a positive constant independent of the complex variable $z$ and the positive integral variable $n$, while $\varepsilon$ is such a constant supposed (as usual) to be arbitrarily small, all these constants being not necessarily the same at each occurrence.

\section{Lemmas}

Lemma 1. Let $s_{n}(n=0,1,2, \ldots)$ be a sequence such that

$$
F(z)=s_{0} z+\frac{s_{1}}{2} z^{2}+\ldots+\frac{s_{n}}{n+1} z^{n+1}+\ldots,
$$


has a circle of convergence $|z|=1$. Then a necessary and sufficient condition for $s_{n} \rightarrow S(l)$ is

$$
\int_{l=1=1-n^{-1}} \frac{F(z)-S \log (1-z)^{-1}}{(1-z) z^{n+1} \log (1-z)^{-1}} d z=o(\log n), n \rightarrow \infty .
$$

Proof. Firstly, the above integral is

$$
\int_{|z|=1-n^{-1}} \frac{\sum_{0}^{\infty}\left(\sum_{v=0}^{n} \frac{s_{v}-S}{v+1}\right) z^{n+1}}{z^{n+1} \log (1-z)^{-1}} d z=2 \pi i \sum_{v=0}^{n} \frac{s_{v}-S}{v+1}
$$

by Cauchy's theorem. Secondly, the right-hand member being $o(\log n)$ is equivalent to $s_{n} \rightarrow S(l)$ by definition (1). These two statements together prove Lemma 1.

Lemma 2. Let $F(z)$ be defined as in Lemma 1. Then

$$
s_{n}=o(n \log n) \text { implies } F\left(\overline{1-\frac{1}{n}} e^{i \theta}\right)=o(n \log n), n \rightarrow \infty .
$$

Proof. If $z=\left(1-\frac{1}{n}\right) e^{i \theta}$, then

$$
\frac{1}{n}\left|F\left(\overline{1-\frac{1}{n}} e^{i \theta}\right)\right| \leqq(1-|z|)\left(\sum_{0}^{v_{0}-1}+\sum_{v_{0}}^{\infty}\right) \frac{\left|s_{v}\right|}{v+1}|z|^{v+1},
$$

where $v_{0}$ is chosen so that $\frac{\left|s_{v}\right|}{v+1}<\varepsilon \log v$. for $v \geqq v_{0}$, on the assumption $s_{n}=o(n \log n)$. With this assumption, we have then

$$
\begin{aligned}
& \frac{1}{n}\left|F\left(1-\frac{1}{n} e^{i \theta}\right)\right|<(1-|z|) \sum_{0}^{v_{0}-1} \frac{\left|s_{v}\right|}{v+1}|z|^{v+1}+\varepsilon(1-|z|) \sum_{v_{0}}^{\infty}|z|^{v+1} \log v \\
& =(1-|z|) \sum_{0}^{v_{0}-1} \frac{\left|s_{v}\right|}{v+1}|z|^{v+1}+\varepsilon|z|^{v_{0}+1} \log v_{0}+\varepsilon \sum_{v_{0}}^{\infty}|z|^{v+2} \log \left(1+\frac{1}{v}\right) \\
& <K+\varepsilon \sum_{v_{0}}^{\infty} \frac{|z|^{v+2}}{v} \\
& =k^{1}-\varepsilon|z|^{2} \log (1-|z|)=k^{1}-\varepsilon\left(1-\frac{1}{n}\right)^{2} \log 1 / n .
\end{aligned}
$$

The last inequality is the one required.

Lemma 3. Let $F(z)$ be defined as in Lemma 1. Also, for $z_{0}$ and $z_{1}$ such that $0<\left|z_{0}\right|<1$ and $0<|z|<1$, let us define

$$
\begin{aligned}
& F_{1}(z)=F_{1}\left(z_{0}\right)+\int_{z_{0}}^{z} \frac{F(w)}{w^{n+1}} d w \\
& F_{2}(z)=F_{2}\left(z_{0}\right)+\int_{z_{0}}^{z} F_{1}(w) d w
\end{aligned}
$$

E.M.S.-D 
choosing some path of integration within $|z|=1$. Then $s_{n}=o(n \log n), n \rightarrow \infty$, implies

$$
F_{1}\left(\overline{1-\frac{1}{n}} e^{i \theta}\right)=o(n \log n), F_{2}\left(\overline{1-\frac{1}{n}} e^{i \theta}\right)=o(\log n) .
$$

Proof. The first relation of (8) follows from a combined application to (6) of Lemma 2 and the maximum-modulus principle.

To prove the second relation of (8), we substitute for $F(z)$ in (6) its expression as a power series and get, integrating term by term,

$$
\begin{aligned}
F_{1}(z) & =F_{1}\left(z_{0}\right)+\int_{z_{0}}^{z}\left(\sum_{v=0}^{\infty} \frac{s_{v}}{v+1} w^{v+1}\right) w^{-n-1} d w \\
& =k+\sum_{v \geqq 0, v \neq n-1} \frac{s_{v}}{(v+1)(v-n+1)} z^{v-n+1}+\frac{s_{n-1}}{n} \log z .
\end{aligned}
$$

Once more integrating term by term, we get $F_{2}(z)$ in the form

$$
\left.\begin{array}{rl}
F_{2}(z) & =F_{2}\left(z_{0}\right)+K\left(z-z_{0}\right)+\frac{s_{n-1}}{n}(z \log z-z)-\frac{s_{n-1}}{n}\left(z_{0} \log z_{0}-z_{0}\right) \\
& -\frac{s_{n-2}}{n-1}\left(\log z-\log z_{0}\right)+\sum_{\substack{v \geqq 0, v \neq n-1 \\
v \neq n-2}} \frac{s_{v}}{(v+1)(v-n+1)(v-n+2)} z^{v-n+2} \cdot
\end{array}\right\}
$$

Since $s_{n}=o(n \log n)$ we can choose $v_{0}$ so that

$$
\frac{\left|s_{v}\right|}{v-1}<\varepsilon \log v \text { for } v \geqq v_{0}, \frac{\left|s_{v}\right|}{v+1}<M \text { for } v<v_{0} \text {. }
$$

We then get from (9), taking $z=\left(1-\frac{1}{n}\right) e^{i \theta}$ and letting $n \rightarrow \infty$,

$$
\begin{aligned}
& F_{2}\left(1-\frac{1}{n} e^{i \theta}\right) \\
& =O(1)-o(\log n)+\left(\sum_{v=0}^{v_{0}-1}+\sum_{v=v_{0}}^{n-3}+\sum_{v=n}^{\infty}\right) \frac{s_{v}}{(v+1)(v-n+1)(v-n+2)} z^{v-n+2} \\
& =o(\log n)+\Sigma_{1}+\Sigma_{2}+\Sigma_{3} \text { (say). }
\end{aligned}
$$

Now

Therefore,

$$
|z|^{-n}=\left(1-\frac{1}{n}\right)^{-n}=\left(1+\frac{1}{n-1}\right)\left(1+\frac{1}{n-1}\right)^{n-1}<2 e \quad(n>1) .
$$

$$
\begin{aligned}
\left|\Sigma_{1}\right|<|z| & \sum_{v=0}^{-n} \frac{\left|s_{v}\right|}{v+1} \frac{1}{(n-v-1)(n-v-2)} \\
& <2 e M \sum_{v=0}^{v_{0}-1} \frac{1}{(n-v-1)(n-v-2)}<\frac{2 e M v_{0}}{\left(n-v_{0}\right)\left(n-v_{0}-1\right)}=o\left(\frac{1}{n}\right) .
\end{aligned}
$$


Similarly,

$$
\begin{aligned}
\left|\Sigma_{2}\right| & <2 e \varepsilon \sum_{v=v_{0}}^{n-3} \frac{\log v}{(n-v-1)(n-v-2)} \\
& <2 e \varepsilon \log n\left\{\frac{1}{1.2}+\frac{1}{2.3}+\ldots+\frac{1}{\left(n-v_{0}-1\right)\left(n-v_{0}-2\right)}\right\} \\
& <2 e \varepsilon \log n .
\end{aligned}
$$

Lastly, in the same way,

$$
\begin{aligned}
\left|\Sigma_{3}\right| & <2 e \varepsilon \sum_{v=n}^{\infty} \frac{\log v}{(v-n+1)(v-n+2)} \\
& =2 e \varepsilon\left(\sum_{v=0}^{n}+\sum_{v=n+1}^{\infty}\right) \frac{\log (v+n)}{(v+1)(v+2)} \\
& <2 e \varepsilon\left\{\log 2 n \sum_{v=0}^{n} \frac{1}{(v+1)(v+2)}+\sum_{v=n+1}^{\infty} \frac{\log (2 v-1)}{(v+1)(v+2)}\right\} \\
& <2 e \varepsilon\{\log 2+\log n+o(1)\} .
\end{aligned}
$$

Using (11), (12) and (13) in (10), we get the second relation of (8).

\section{Theorems}

Theorem 1. Suppose that a sequence $s_{n}(n=0,1,2, \ldots)$ is such that $s_{n}=o(n \log n)$ and hence

$$
F(z)=\sum_{0}^{\infty} \frac{s_{n}}{n+1} z^{n+1}
$$

has circle of convergence $|z|=1$. If then $\eta$ is any positive number, however small, a necessary and sufficient condition for $s_{n} \rightarrow S(l)$ is as follows:

$$
\begin{aligned}
\int_{\theta=-\eta}^{\theta=\eta}\left\{F(z)-S \log (1-z)^{-1}\right\} & \\
& \times\left\{\frac{1}{(1-z) \log (1-z)^{-1}}+c_{0}(1-z)+c_{1}(1-z)^{2}\right\} \frac{d z}{z^{n+1}}=o(\log n),
\end{aligned}
$$

where

$$
z=\left(1-\frac{1}{n}\right) e^{i \theta}, c=c_{0}(\eta) \text { and } c_{1}=c_{1}(\eta)
$$

are constants independent of $n$.

Proof. It is obvious that, if we suppose the sequence $s_{n}$ replaced by $s_{n}-S$, we shall have $S=0$, the condition $s_{n}=o(n \log n)$ remaining unaffected. We shall make this supposition for simplicity. Then, by Lemma 1 , a condition necessary and sufficient for $s_{n} \rightarrow 0(l)$ is

$$
\int_{|z|=1-n^{-1}} \frac{F(z)}{(1-z) \log (1-z)^{-1}} \frac{d z}{z^{n+1}}=o(\log n) .
$$


On the other hand, whatever be the constants $c_{0}$ and $c_{1}$, we have

$$
\begin{array}{rl}
\int_{I=1}=1-n^{-1} & F(z)\left\{c_{0}(1-z)+c_{1}(1-z)^{2}\right\} \frac{d z}{z^{n+1}} \\
& =\int_{|=|=1-n^{-1}}\left\{\sum_{0}^{\infty} \frac{s_{v}}{v+1} z^{v+1}\right\}\left\{c_{0}+c_{1}-\left(c_{0}+2 c_{1}\right) z+\left(c_{0}+c_{1}\right) z^{2}\right\} \frac{d z}{z^{n+1}} \\
& =\left(c_{0}+c_{1}\right) \frac{s_{n-1}}{n}-\left(c_{0}+2 c_{1}\right) \frac{s_{n-2}}{n-1}-\left(c_{0}+c_{1}\right) \frac{s_{n-3}}{n-2}=o(\log n)
\end{array}
$$

by hypothesis. From (15) and (16) we see that a condition necessary and sufficient for $s_{n} \rightarrow 0(l)$ is

$$
\int_{C} F(z)\left\{\frac{1}{(1-z) \log (1-z)^{-1}}+c_{0}(1-z)+c_{1}(1-z)^{2}\right\} \frac{d z}{z^{n+1}}=o(\log n)
$$

where $C$ is the circle $|z|=1-n^{-1}$ and $c_{0}, c_{1}$ are any constants. Comparing the above condition with (14) for $S=0$, we note that it suffices to prove:

$$
\left.\begin{array}{l}
\int_{C^{\prime}} F(z)\left\{\frac{1}{(1-z) \log (1-z)^{-i}}+c_{0}(1-z)+c_{1}(1-z)^{2}\right\} \frac{d z}{z^{n+1}}=o(\log n) \\
\text { where } \\
\qquad C^{\prime} \text { is the arc: } z=\left(1-\frac{1}{n}\right) e^{i \theta}, \eta \leqq \theta \leqq 2 \pi-\eta,
\end{array}\right\}
$$

and $c_{0}, c_{1}$ are constants suitably chosen.

To determine such a choice of $c_{0}, c_{1}$ we write

and make

$$
Q(z)=\frac{1}{(1-z) \log (1-z)^{-1}}+c_{0}(1-z)+c_{1}(1-z)^{2},
$$

$$
Q\left(e^{i \eta}\right)=Q\left(e^{-i \eta}\right)=0
$$

Obviously $c_{0}, c_{1}$ are determined uniquely and independently of $n$ by means of (18). Further, if we write

then, by (18),

$$
z_{1}=r e^{i \eta}, z_{2}=r e^{-i \eta} \text { where } r=1-\frac{1}{n}
$$

$$
\frac{Q\left(r e^{i \eta}\right)}{(r-1) e^{i \eta}}=\frac{Q\left(z_{1}\right)}{z_{1}-e^{i \eta}} \rightarrow Q^{\prime}\left(e^{i \eta}\right) \text { as } z_{1} \rightarrow e^{i \eta} \text { or } r \rightarrow 1-0 \quad(n \rightarrow \infty),
$$

this relation being true also with $z_{2}$ and $-\eta$ in place of $z_{1}$ and $\eta$ respectively. Letting $r \rightarrow 1-0$, we get then

$$
\left.\begin{array}{ll}
\left|Q^{\prime}\left(z_{1}\right)\right|<M,\left|Q\left(z_{1}\right)\right|<M(1-r)=M n^{-1} & (n \rightarrow \infty), \\
\left|Q^{\prime}\left(z_{2}\right)\right|<N,\left|Q\left(z_{2}\right)\right|<N(1-r)=N n^{-1} & (n \rightarrow \infty)
\end{array}\right\}
$$

Having defined $Q(z)$ by the condition (18), we proceed to complete the 
proof by establishing (17) in the form

$$
\int_{z_{1}}^{z_{2}} \frac{F(z)}{z^{n+1}} Q(z) d z=o(\log n)
$$

where $z_{1}$ and $z_{2}$ are given by (19) and the path of integration is the circle $|z|=1-n^{-1}$. Integrating twice by parts the integral of (21), and using the definitions of $F_{1}(z), F_{2}(z)$ in (6), (7) respectively, we get

$$
\left|\int_{z_{1}}^{z_{2}} \frac{F(z)}{z^{n+1}} Q(z) d z\right| \leqq\left|\left[F_{1}(z) Q(z)\right]_{z_{1}}^{z_{2}}\right|+\left|\left[F_{2}(z) Q^{\prime}(z)\right]_{z_{1}}^{z_{2}}\right|+\left|\int_{z_{1}}^{z_{2}} F_{2}(z) Q^{\prime \prime}(z) d z\right| \text {. }
$$

In the first and the second terms of the right-hand member, we use the estimates for $F_{1}(z)$ and $F_{2}(z)$ in (8) along with the estimates for $Q\left(z_{1}\right), Q\left(z_{2}\right)$ and $Q^{\prime}\left(z_{1}\right)$, $Q^{\prime}\left(z_{2}\right)$ given by $(20)$. The result is

$$
\begin{aligned}
\left|\int_{z_{1}}^{z_{2}} \frac{F(z)}{z^{n+1}} Q(z) d z\right| & \leqq o(\mathrm{n} \log n) O\left(n^{-1}\right)+o(\log n)+\left|\int_{z_{1}}^{z_{2}} F_{2}(z) Q^{\prime \prime}(z) d z\right| \\
& =o(n \log n) O\left(n^{-1}\right)+o(\log n)+o(\log n) .
\end{aligned}
$$

The passage from (22) to the last step is justified by the fact that, on the path of integration, $Q^{\prime \prime}(z)$ is bounded while $F_{2}(z)=o(\log n)$ by (18). And the last step leads obviously to (21), thus completing the proof.

Theorem 2. Suppose that a sequence $s_{n}(n=0,1,2, \ldots)$ is such that

$$
\text { (i) } s_{n}=o(n \log n), \quad n \rightarrow \infty
$$

and therefore $F(z)=\sum_{0}^{\infty} \frac{s_{n}}{n+1} z^{n+1}$ has circle of convergence $|z|=1$. Suppose also that

$$
\text { (ii) } \frac{F(z)}{\log (1-z)^{-1}}=O(1) \text { uniformly when }|z|<1, \mid \text { am } z \mid \leqq \eta \text {. }
$$

Then $s_{n} \rightarrow S(l)$ whenever $s_{n} \rightarrow S(L)$.

Proof. As in the proof of Theorem 1, we may take $S=0$. Starting then with the assumption $s_{n} \rightarrow 0(L)$, we have to prove that $s_{n} \rightarrow 0(l)$ on the further hypotheses (i), (ii) of Theorem 2. We know (by Theorem 1) that, on hypothesis (i), a necessary and sufficient condition for $s_{n} \rightarrow 0(l)$ is (14) with $S=0$. Thus in order to conclude that $s_{n} \rightarrow 0(l)$ it only remains to prove that hypothesis (ii) and $s_{n} \rightarrow 0(L)$ together imply (14) with $S=0$, or more particularly,

$$
\begin{aligned}
& I_{1}=\int_{\theta=0}^{\theta=n} \frac{F(z)}{(1-z) \log (1-z)^{-1}} \frac{d z}{z^{n+1}}=o(\log n)\left[z=\left(1-\frac{1}{n}\right) e^{i \theta}, n \rightarrow \infty\right], \\
& I_{2}=\int_{\theta=0}^{\theta=\eta} F(z)\left\{c_{0}(1-z)+c_{1}(1-z)^{2}\right\} \frac{d z}{z^{n+1}}=O(1)\left[z=\left(1-\frac{1}{n}\right) e^{i \theta}, n \rightarrow \infty\right] .
\end{aligned}
$$


To prove (25) we observe that, for $z=\left(1-\frac{1}{n}\right) e^{i \theta}$,

$$
\begin{aligned}
\left|I_{2}\right| & \leqq\left|c_{0}\right| \int_{0}^{n}\left|F(z)\left\|1-z\left|\left(1-\frac{1}{n}\right)^{-n} d \theta+\right| c_{1}\left|\int_{0}^{n}\right| F(z)\right\| 1-z\right|^{2}\left(1-\frac{1}{n}\right)^{-n} d \theta \\
& =I_{21}+I_{22} \text { (say). }
\end{aligned}
$$

Here the integrand of $I_{21}$ is bounded as $n \rightarrow \infty$. For,

$$
\left(1-\frac{1}{n}\right)^{-n}<2 e \quad(n>1)
$$

as we have already seen, and, on account of (23),

$$
\begin{aligned}
|F(z)| 1-z \mid & <k\left|(1-z) \log (1-z)^{-1}\right| \\
& <k\left(1+\frac{1}{1.2}+\frac{1}{2.3}+\ldots\right)=2 k .
\end{aligned}
$$

Therefore, in (26),

$$
I_{21}=O(1), I_{22}=o(1), n \rightarrow \infty
$$

whence (25) follows at once.

Thus we have finally to prove (24). To this end, we write the integral in (24) as

$$
I_{1}=\left\{\int_{\theta=0}^{\theta=\frac{1}{\log n}}+\int_{\theta=\frac{1}{\log n}}^{n}\right\} \frac{F(z)}{(1-z) \log (1-z)^{-1}} \frac{d z}{z^{n+1}}=I_{11}+I_{12} \text { (say) }
$$

where

and $n_{0}$ is chosen so that

$$
z=\left(1-\frac{1}{n}\right) e^{i \theta}, \quad n \geqq n_{0}
$$

$$
\left|\frac{F(z)}{\log (1-z)^{-1}}\right|<\varepsilon \text { for } z=\left(1-\frac{1}{n_{0}}\right) e^{i \theta}, \quad 0 \leqq \theta \leqq \frac{1}{\log n_{0}}<\eta .
$$

A choice of $n_{0}$ as in (28) is possible because of our initial assumption $s_{n} \rightarrow 0(L)$ and its implication corresponding to (5). Since (28) is true a fortiori with $n_{0}$ replaced by $n$, we see that, in (27),

$$
\begin{aligned}
\left|I_{11}\right|<\varepsilon \int_{0}^{\frac{1}{\log n}} \frac{\left(1-\frac{1}{n}\right)^{-n}}{1-\left(1-\frac{1}{n}\right) e^{i \theta} \mid} d \theta \\
\quad<2 \varepsilon e \int_{0}^{\frac{1}{\log n}} \frac{n d \theta}{\left\{1+4 n(n-1) \sin ^{2} \frac{\theta}{2}\right\}}<2 \varepsilon e \int_{0}^{\frac{1}{\log n}} \frac{N n d \theta}{\left(N^{2}+n^{2} \theta^{2}\right)^{\frac{1}{2}}},
\end{aligned}
$$

$N$ being a constant independent of $n$ and $\theta$. Hence, integrating the last 
integral, we obtain

$$
\begin{aligned}
\left|I_{11}\right| & <2 \varepsilon e N \log \left\{\frac{n}{N \log n}+\left(1+\frac{n^{2}}{N^{2} \log ^{2} n}\right)^{\frac{1}{2}}\right\} \\
& <2 \varepsilon e N \log 2\left(1+\frac{n^{2}}{N^{2} \log ^{2} n}\right)^{\frac{1}{t}} \\
& =2 \varepsilon e N\left\{\log 2+\log \frac{n}{N \log n}+O\left(\frac{\log ^{2} n}{n^{2}}\right)\right\} \\
& =2 \varepsilon e N\{\log n+o(\log n)\} .
\end{aligned}
$$

We treat similarly $I_{12}$ in (27), but using (23) instead of (28), and get the result:

$$
\begin{aligned}
\left|I_{12}\right| & <2 k e \int_{\frac{1}{\log n}}^{n} \frac{N n d \theta}{\left\{N^{2}+n^{2} \theta^{2}\right\}^{\frac{1}{2}}} \\
& <2 k N e \int_{\frac{1}{\log n}}^{n} \frac{d \theta}{\theta}=2 k N e(\log \eta+\log \log n)=o(\log n) .
\end{aligned}
$$

Now we use (29) and (30) in (27) to obtain (24) which, along with (25) earlier proved, concludes the proof as already observed.

The following deduction from Theorem 2 is obvious.

Corollary. If $s_{n}(n=0,1,2, \ldots)$ is a sequence such that

$$
\begin{aligned}
s_{n} & =o(n \log n), \\
\frac{F(z)}{\log (1-z)^{-1}}= & \frac{\sum_{0}^{\infty} \frac{s_{n}}{n+1} z^{n+1}}{\log (1-z)^{-1}} \rightarrow S
\end{aligned}
$$

as $z \rightarrow 1$ along any path within $|z|=1$, then $s_{n} \rightarrow S(l)$.

The author is indebted to Prof. C. T. Rajagopal for his kind help in the preparation of this note.

\section{REFERENCES}

(1) G. H. HARDY, Divergent Series (Oxford, 1949).

(2) K. Ishiguro, On the summability methods of logarithmic type, Proc. Japan Acad., 38 (1962), 703-705.

(3) A. C. Offord, On the summability of power series, Proc. London Math. Soc. (2), 33 (1932), 467-480.

(4) M. S. Rangachari and Y. Sitaraman, Tauberian theorems for logarithmic summability (L), Tôhoku Math. J. (2), 16 (1964), 257-269.

Ramanujan Institute of Mathematics

UNIVERSITY OF MADRAS

MADRAS-5, INDIA 